\title{
Mission en temps de guerre : Alfred Métraux dans le Pilcomayo
}

Misión en tiempos de guerra: Alfred Métraux en el Pilcomayo

War-time Mission: Alfred Métraux in the Pilcomayo

\section{Lorena I. Córdoba}

\section{(2) OpenEdition}

Journals

Édition électronique

URL : https://journals.openedition.org/jsa/14806

DOI : 10.4000/jsa. 14806

ISSN : 1957-7842

\section{Éditeur}

Société des américanistes

Édition imprimée

Date de publication : 31 décembre 2016

Pagination : 45-73

ISSN : 0037-9174

\section{Référence électronique}

Lorena I. Córdoba, « Mission en temps de guerre : Alfred Métraux dans le Pilcomayo », Journal de la Société des américanistes [En ligne], 102-2 | 2016, mis en ligne le 26 janvier 2017, consulté le 04

septembre 2022. URL : http://journals.openedition.org/jsa/14806 ; DOI : https://doi.org/10.4000/jsa 14806 


\title{
Mission en temps de guerre: Alfred Métraux dans le Pilcomayo
}

\author{
Lorena I. CóRDOBA *
}

Ce travail analyse la relation entre Alfred Métraux et les Indiens Toba et Pilagá de l'ouest de Formosa (Argentine) en 1933. Il décrit les circonstances sociopolitiques singulières des terrains du jeune anthropologue: les problèmes suscités par la difficile situation économique de l'université de Tucumán, la guerre du Chaco opposant la Bolivie au Paraguay (1932-1935), l'inévitable relation avec les missions anglicanes de la South American Missionary Society et la situation frontalière très tendue entre l'Argentine et le Paraguay. En entrecroisant lettres personnelles, textes publiés et sources orales et écrites, on propose d'analyser le contexte général des premiers terrains ethnographiques de Métraux dans le Gran Chaco. [Mots-clés: Métraux, missionnaires, guerre, Indiens, Gran Chaco.]

Misión en tiempos de guerra: Alfred Métraux en el Pilcomayo. Este trabajo analiza la relación entre Alfred Métraux y los indígenas tobas y pilagás del Oeste formoseño en 1933. Se detallan las peculiares circunstancias sociopolíticas que enmarcan las campañas de terreno del joven antropólogo: los problemas suscitados por la penosa situación económica en la Universidad de Tucumán, la Guerra del Chaco entre Bolivia y Paraguay (1932-1935), la ineludible relación con las misiones anglicanas de la South American Missionary Society y la tensa situación fronteriza entre Argentina y Paraguay. A través del entrecruzamiento de cartas personales, textos publicados y fuentes orales y escritas, se propone analizar pues el contexto general de las primeras campañas etnográficas de Métraux en el Gran Chaco. [Palabras clave: Métraux, Misioneros, Guerra, Indígenas, Gran Chaco.]

War-time Mission: Alfred Métraux in the Pilcomayo. The paper analyses the relationship between Alfred Métraux and the Toba and Pilagá peoples of Western Formosa (Argentina) during 1933. The peculiar sociopolitical circumstances that encompassed Métraux's fieldwork are detailed: the logistical problems due to the feeble economic situation of University of Tucumán, the Chaco War between Bolivia and Paraguay (1932-1935), the inescapable relationship with the Anglican settlements of the South American Missionary Society and the tense situation on

* Conicet, Universidad de Buenos Aires (Argentine)/Centro de Investigaciones Históricas y Antropológicas (Bolivie) [lorecordoba@gmail.com]. 
the national frontier between Argentina and Paraguay. By combining personal letters, published texts and oral and written sources, the paper aims to reconstruct the general context of the first ethnographic experiences of Métraux in the Gran Chaco. [key words: Métraux, Missionaries, War, Indigenous groups, Gran Chaco.]

À mon sens, le seul intérêt, la seule valeur de cette terre déshéritée est représentée par les admirables tribus indiennes qui ont réussi à se cramponner et à y conserver presque intacte l'image de leur vie passée. Nulle tribu indienne n'a été plus calomniée, nulle n'a été traitée avec plus de cruauté et nulle n'a conquis à un plus haut degré la sympathie et l'affection de l'ethnographe qui a voulu vivre de leur vie. Métraux 2013 [1937], p. 433.

Le 8 janvier 1933, le jeune Alfred Métraux écrit à son collègue et ami Rodolfo Schreiter, de l'université de Tucumán: « Dites à ces Messieurs de Tucumán que l'ethnographie n'est pas toujours une aimable partie de plaisir et que l'envie de voir les Indiens revient relativement cher ${ }^{1}$. » À combien faut-il évaluer cette « relative cherté » du travail de terrain de 1933 chez les Toba et les Pilagá de l'ouest de la région de Formosa? Contrairement à son habitude, Métraux ne tint pas durant cette expédition de journal consignant minutieusement son parcours. Il nous faut donc le reconstruire à partir de ses textes et de sa correspondance, ainsi que des écrits d'autres voyageurs et diverses publications de l'époque.

À cette époque, deux faits historiques marquaient indéfectiblement la vie des « Toba » ou « Toba-Pilagá » et affectèrent sans doute aussi l'expérience

1. Lettre à Schreiter du 8 janvier 1933, citée in Amenta 2008 (p. 177-178); voir aussi Perilli de Colombres (2006), qui propose que l'expression « ces Messieurs de Tucumán » (" los señores de Tucumán ») se réfère aux dirigeants de l'université, ce qui semble logique compte tenu des autres relations auxquelles l'auteur fait allusion.

2. Appartenant à la famille linguistique guaycurú (dont relèvent également les autres groupes appelés « Toba », les Mocoví et les Pilagá), les « Toba-Pilagá » (pour reprendre la terminologie proposée par Métraux lui-même en 1937) apparaissent parfois également dans la bibliographie sous la dénomination de « Toba de l'ouest », « Toba ñachilamole\#ek» ou «Toba de Sombrero Negro ». Ils s'auto-désignent « Toba» ou qom 'le\#tek, qom- étant un morphème qui (en conjonction avec les suffixes pluralisateurs, - pi et le masculin, -le\#tek ou le féminin -lashé) renvoie génériquement aux idées de " personne ", « humanité », " gens ». Ils appellent les Pilagá ta'yiñile\#ek (« gens de l'aval ») et ces derniers les appellent en retour ñachilamole\#ek (ñachi : " fleuve », lamo: «tronc », le\#tek: « gens »). Comme dans cette région le cours du fleuve Pilcomayo est encaissé entre deux rives escarpées, par contraste avec la zone sud-orientale où il se jette dans le marais La Estrella, une traduction plus juste de l'ethnonyme serait: " gens du Pilcomayo encaissé » (Braunstein et Córdoba 2008). Métraux (1937, p. 174) considère les Pilagá comme un sous-groupe des Toba et forge l'ethnonyme « Toba-Pilagá » en vertu des ressemblances culturelles et linguistiques entre les deux groupes: «Les Indiens de Sombrero Negro se considèrent comme des Toba. Ni 
de l'ethnographe qui leur rendit visite pendant plusieurs mois en 1933. D'un côté, la présence des missionnaires anglicans, qui vivaient avec eux depuis trois ans et avec lesquels Métraux devait également s'habituer à interagir. Et d'autre part - vu 1'emplacement géographique de Misión El Toba, de Misión Pilagá et des divers établissements indigènes que visita cette année-là le Suisse sur les rives du Pilcomayo - les débuts de cet événement majeur que fut la guerre du Chaco, qui opposa la Bolivie au Paraguay entre 1932 et 1935.

La campagne chez les Pilagá et les Toba se déroula entre décembre 1932 et avril 1933. Cela faisait un peu moins de quatre ans que Métraux dirigeait l'Institut d'ethnologie et le musée ethnographique de l'université de Tucumán, où il avait été recruté sur la recommandation de son maître Paul Rivet, directeur du musée du Trocadéro ${ }^{3}$. Peu de mois après son arrivée, il organisa sa première expédition ethnographique: de février à juin 1929, il visita les Chiriguano et les Chané du sud de la Bolivie et du nord de l'Argentine ${ }^{4}$. L'année suivante il réalisa une autre expédition avec le conservateur du musée d'Histoire naturelle, Rodolfo Schreiter, qui devint bientôt son ami et avec qui il fit des fouilles archéologiques à La Candelaria (Salta) pour identifier les vestiges d'une culture qu'ils baptiseraient de ce nom ${ }^{5}$. L'année d'après, Métraux entreprit une nouvelle expédition ethnographique financée par l'université de Tucumán, en janvier et février de 1931, pour rendre visite aux Chipaya de Carangas; et enfin, en juin de cette même année, il fit le déplacement jusqu'au village bolivien de Desaguadero ${ }^{6}$.

\section{Deux voyages chez les Toba et les Pilagá}

En 1932, Métraux se trouve à Buenos Aires. Les lettres qu'il envoie à Schreiter nous révèlent ses plans ethnologiques. Après de nombreux efforts, il parvient à concrétiser deux projets de voyage chez les Toba: une première campagne,

par leur langue, ni par leurs coutumes ils ne diffèrent des "gens d'en bas", c'est-à-dire des Pilagá de Descanso avec lesquels la plupart sont apparentés. Il existe cependant entre le toba de Sombrero Negro et celui de Descanso, une petite différence dialectale: à Sombrero Negro le $s$ initial s'est mué en $h$ aspiré. » À l'heure actuelle, même s'ils continuent à partager un certain univers culturel et même s'il existe des circuits de réciprocité ou des relations d'affinité et de consanguinité entre les Toba de l'ouest et les Pilagá de la région de Las Lomitas, chacun se considère comme un groupe à part et la dénomination « TobaPilagá » n'est aucunement reconnue comme renvoyant à un groupe ethnique. Il y a de nos jours quelque deux mille Toba dans le département de Bermejo, à environ cinquante kilomètres du bourg de Ingeniero Juárez (De la Cruz 1995; Gordillo 1999a; Arenas 2003; Córdoba et Braunstein 2008; Gómez C. 2011; Gómez M. 2011).

3. Perilli de Colombres 2006, p. 146. Pour une analyse des relations entre Alfred Métraux et Paul Rivet, voir l'excellente biographie de ce dernier publiée par Laurière (2008).

4. Bossert et Villar 2007.

5. Métraux 1930.

6. Sendón 2009. 


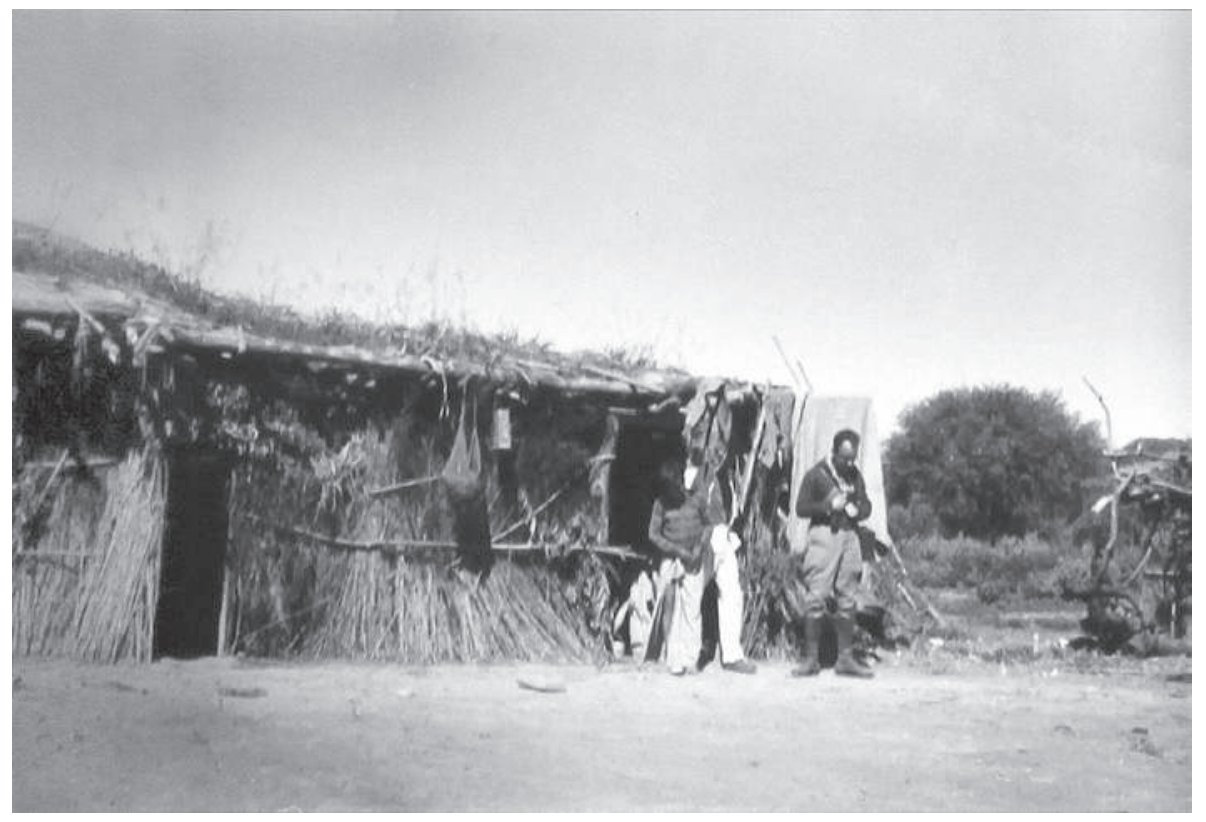

Fig. 1 - « Indiens, huttes et A. M. », photo par Guy Métraux, 1939

(Guy Métraux C musée du quai Branly - Jacques Chirac).

de décembre 1932 à avril 1933, et une seconde de septembre à octobre de cette même année. Cependant il n'existe aucune trace précise de ses itinéraires. Les notes de terrain de Métraux ne démarrent qu'au début de 1935 et ce n'est qu'à partir de là qu'on peut retracer pas à pas son séjour chez les Toba en 1939 en compagnie de son frère Guy. Pour reconstituer ses tout premiers voyages, on doit en revanche se contenter de sources indirectes: les lettres que le jeune ethnographe envoyait depuis le terrain à Schreiter ou à son amie Yvonne Oddon, ses publications dans le Journal de la société des américanistes (JSA), dans le journal argentin La Prensa et ses brèves notes publiées en anglais dans la revue des religieux anglicans qui travaillaient avec les Toba (South American Missionary Society Magazine ou SAMSM). C'est tout ce qu'il nous reste pour reconstituer le contexte qui expliquerait, ne serait-ce que partiellement, ses plaintes récurrentes contre la chaleur, les insectes, la puanteur, la monotonie du régime ou le manque cruel de ressources économiques.

Fin décembre 1932, Métraux arrive à Las Lomitas et en profite pour commen-

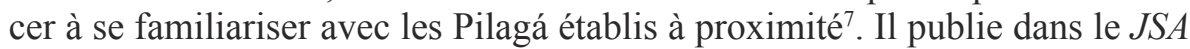

7. En réalité, selon ses cahiers de terrain archivés dans le FAM, le travail avec quelques Toba rencontrés dans la ville de Formosa avait déjà commencé avant même son arrivée à Las Lomitas, le 19 décembre 1932. Dans une lettre, il précise: «Je me serais ennuyé 
une brève note de recherche intitulée « Nouvelles de la mission A. Métraux », en date du 8 janvier, où il raconte les succès et les difficultés qu'il rencontre en compagnie d'Elizabeth Dijour'. Ils partent à cheval jusqu'à la région marécageuse d'Estero Patiño en route vers les Pilagá, et au bout de dix jours rencontrent un petit groupe décimé par une épidémie de variole: « nous avons vu là des scènes horribles », note-t-il $1^{9}$. Jadis une des tribus les plus importantes du territoire argentin, les Pilagá ne sont plus guère qu'une trentaine. À Labagan (Pozo Navagán), capitale des Pilagá, les membres de l'expédition rencontrent le chef Garcete, traversent le Pilcomayo et sur le territoire paraguayen, alors que les hostilités battent leur plein, parviennent à se constituer une belle collection de tissages maká ainsi qu'à inventorier plus de deux cents jeux de ficelle. Du côté argentin, ils passent ensuite par Fortín Salazar, Fortín Lugones et Fortín Descanso - toponymie donnant une bonne idée de l'importance du conflit à l'échelle régionale -, où ils comptent travailler quelques jours avec le grand chef pilagá Lagadik. Le sort leur est cependant une fois de plus adverse, non tant à cause de la variole que faute d'avoir trouvé de bons traducteurs, ce qui leur interdit de recueillir des récits en langue vernaculaire ${ }^{10}$. Frustré, Métraux relate à Schreiter ses sentiments sur ces premiers jours:

La première partie de mon voyage a été un succès complet, si on s'en tient au nombre et à la qualité des objets collectés, mais à part ça je dois bien confesser que j'ai souffert de grandes désillusions. Les Indiens sont en train de disparaître, décimés par la variole noire et c'est à grand renfort de peines que j'ai pu trouver quelques rares campements [tolderías] où agonisent un petit nombre d'Indiens. À ce rythme, d'ici un an, il n'y aura pratiquement plus d'Indiens dans cette région ${ }^{11}$.

Au Fortín Lugones, Métraux relate sa rencontre avec un fameux guérillero paraguayen, Plácido Jara, et ses " macheteros de la muerte », qui participaient

à mourir si je n'avais pas rencontré quelques Indiens Toba avec lesquels je me suis lié d'amitié et avec lesquels j'ai passé mes journées à parler de la crise et à étudier un peu leur langue qui est plus rêche et difficile que le switzertutsch, ce qui n'est pas peu dire » (citée in Amenta 2008, p. 166). Tout au long de centaines et de centaines de pages, les cahiers de terrain de Métraux rendent compte de son travail au cours de ces premières années: il recueille un vaste vocabulaire, une bonne quantité de mythes pilagá, toba et wichí, ainsi que des fragments d'information sur la parenté et l'organisation sociale. Précisons également que ces premiers Toba rencontrés à Formosa ne sont pas les mêmes que ceux qui habitent Misión El Toba ou les établissements environnants, où il travaillera intensivement par la suite.

8. Métraux 1933d. Envoyée par le musée du Trocadéro de Paris, Dijour, une étudiante de Boas, publiera un article sur les Wichí dans le JSA (Dijour 1933). Sur son travail de terrain et l'opinion personnelle de Métraux sur sa personne, on peut se référer à sa correspondance avec Yvonne Oddon (Yale).

9. Métraux 1933c, p. 204.

10. Métraux 1937, p. 172. Pour une analyse historique de l'emplacement et du fonctionnement de ces fortins, voir Beck 2005.

11. Lettre à Schreiter du 8 janvier 1933, citée in Amenta 2008, p. 177-178. 
alors activement à la guerre de l'autre côté du Pilcomayo ${ }^{12}$. La lettre contient également d'amères récriminations contre la chaleur du Chaco qui devient insupportable, contre les chevauchées sous un soleil de plomb dans les marais et bien sûr contre les moustiques « qui rendent les gens fous, mais qui moi, en raison de ma puanteur naturelle, ne me dérangent pas trop ${ }^{13} »$. Mais il ne s'agit pas seulement d'obstacles naturels. Les Indiens sont décimés, il n'y a pas de bons interprètes, la variole imprègne les objets qu'il collecte et l'état des chemins est calamiteux :

Demain ou après-demain je partirai pour le Fortín Descanso en espérant avoir plus de chance avec les Indiens. Si je trouve là-bas de grands campements [tolderías] et quelques interprètes, j'y resterai jusqu'en mars, sinon j'abandonnerai cette zone ingrate pour me rendre dans une des missions qui, par ici, ne manquent pas $^{14}$.

Après les frustrations de Fortín Descanso, les ethnologues se dirigent vers la mission anglicane El Toba, dans la localité de Sombrero Negro, à l'ouest de la province de Formosa. Par l'entremise du missionnaire écossais John Arnott, Métraux fait la connaissance du jeune chef Kédok (« Tigre ») qui deviendra son principal traducteur, collaborateur et informateur, et qu'il rencontrera à nouveau en octobre 1933 et en $1939^{15}$. L'amitié entre le chef et l'ethnographe est palpable et les récits sur la vie amoureuse du premier seront à l'origine d'un chapitre important de son œuvre: «Avant de quitter la mission de Sombrero Negro, Kédok me fit cadeau d'un insigne appelé nanaagi. C'est une sorte de cravache faite d'une cordelette en laine tressée, entièrement recouverte de grains en porcelaine ou en verre de différentes couleurs et ornée de pompons. Seuls les braves qui avaient tué un ennemi étaient autorisés à porter ce symbole de leur courage ${ }^{16} .{ }^{\prime \prime}$

Le 25 janvier 1933, Métraux remet aux religieux anglicans un document de deux pages qui sera publié dans la revue $S A M S M$ en juillet de la même année ${ }^{17}$. Curieusement intitulé «A remarkable testimony and appeal from an Argentine scientist », le texte commente en peu de mots le chaleureux accueil des Pilagá de Descanso et une anecdote sur la méprise des Indiens qui le prennent pour un

12. Probablement aux alentours de 1937, Métraux produira un petit rapport dactylographié sur le thème, récemment publié (Métraux 2013 [1937]).

13. Lettre à Schreiter du 8 janvier 1933, citée in Amenta 2008, p. 177-178.

14. Lettre à Schreiter du 8 janvier 1933, citée in Amenta 2008, p. 177-178.

15. Le 24 mars 1939 Métraux $(1978$, p. 63) arrive en pleine nuit à la mission et pour ne pas réveiller le missionnaire Alfred Leake, se dirige directement à la maison de Kédoc, qu'il n'avait pas revu depuis six ans: "Tigre vient à moi avec sa démarche traînante; il a laissé pousser sa moustache et les poils de sa barbe, ce qui lui donne un air déplaisant et hirsute. Il m'appelle "Métraux"... Joie de voir les flammes éclairer des peaux brunes dans l'ombre. » 16. Métraux 1937, p. 397 ; voir Métraux 1967.

17. Métraux 1933c. 
missionnaire anglais. Au même moment, les échanges épistolaires nous permettent d'entrevoir les ombres et lumières de quasi cinq mois passés sur le terrain:

Je suis resté volontairement cinq mois sans recevoir et sans écrire de lettre. J'ai justifié mon silence par la sauvagerie des contrées que j'ai parcouru [...] je n'ai jamais été aussi heureux de ma vie. Ce bonheur ne peut être attribué au voyage lui-même, semé de désagréments, d'inquiétudes et qui a exigé de moi un effort physique bien supérieur à celui que j'ai dû fournir dans mes autres expéditions. J'ai peut-être à mon actif près de $1000 \mathrm{~km}$ à cheval ou à mule, sur des chevaux indiens mal sellés avec la plupart du temps le ventre vide et au milieu de marécages et de nuages de moustiques [...] c'est ensuite par loyauté, par esprit de persévérance que je continue à lutter pour mes Indiens. Ils m'ont payé de mes efforts en m'adoptant et en me laissant une autorité dont l'étendue m'étonne [...]. La mission a été couronnée de succès. J'avouerai même maintenant que les résultats obtenus m'ont surpris et ont été de beaucoup supérieurs à ceux que j'avais prévu dans mes estimations les plus optimistes ${ }^{18}$.

On ne sait pas exactement à quelle date Métraux quitta la mission anglicane mais les lettres échangées avec Schreiter nous permettent d'inférer qu'il serait resté encore quelque temps sur le terrain et ne regagna pas son poste à Tucumán avant avril $1933^{19}$.

Début septembre, il réussit à organiser une nouvelle expédition chez les Toba, se rendant en Bolivie avant de passer par Salta et Formosa ${ }^{20}$. À Misión Chaqueña (parmi les Wichí), il rencontre le couple Saint et entreprend de poursuivre avec eux en voiture jusqu'au sud bolivien ${ }^{21}$. Dans une lettre du 3 octobre

\section{Lettre du 27 avril 1933 à Yvonne Oddon (Yale).}

19. Aux alentours de janvier 1933, Métraux écrit à Schreiter: «Je viens d'arriver à la "Misión de los Tobas" après quatre jours de voyage exténuants en compagnie d'une escorte d'Indiens. Je suis installé dans un campement [toldería] où j'occupe tout à la fois les fonctions de chef et de médecin. Je vis complètement seul baigné d'indianité et profite comme vous le voyez d'un grand prestige. Ce sont eux qui m'ont envoyé ici en qualité d'ambassadeur. Je reviendrai sous peu et resterai parmi mes gens jusqu'en avril si les inondations du Pilcomayo ne m'obligent pas à rester plus longtemps » (citée in Amenta 2008, p. 184). Peu après, le 6 février 1933, le lieutenant-colonel Estévez écrit également à Schreiter pour lui dire qu'il a reçu les 490 pesos qu'il est censé mettre à disposition de Métraux (citée in Amenta 2008, p. 185) et ce n'est que le 4 avril que Métraux envoie un télégramme pour solliciter un autre virement de fonds au motif suivant: "Viens seulement de pouvoir traverser marécages Pilcomayo. Santé précaire » (citée in Amenta 2008, p. 192).

20. Lettre à Schreiter du 18 septembre 1933, citée in Amenta 2008, p. 220-221.

21. Le couple Saint, d'origine française, accompagne Métraux dans le Chaco pendant une partie de ses voyages de 1933, et publie dans la Revista de geografía americana l'itinéraire parcouru dans le nord argentin et le sud bolivien. Propriétaires d'une des plus grandes usines de fabrication de boîtes de biscuits d'Argentine, les Saint aident les missions anglicanes par des dons de vêtements et d'aliments. En 1935 le couple arrive à Misión El Toba et y retrouve Kédoc, qu'ils connaissaient grâce aux récits que Métraux leur avait faits deux ans plus tôt (Saint 1936). 


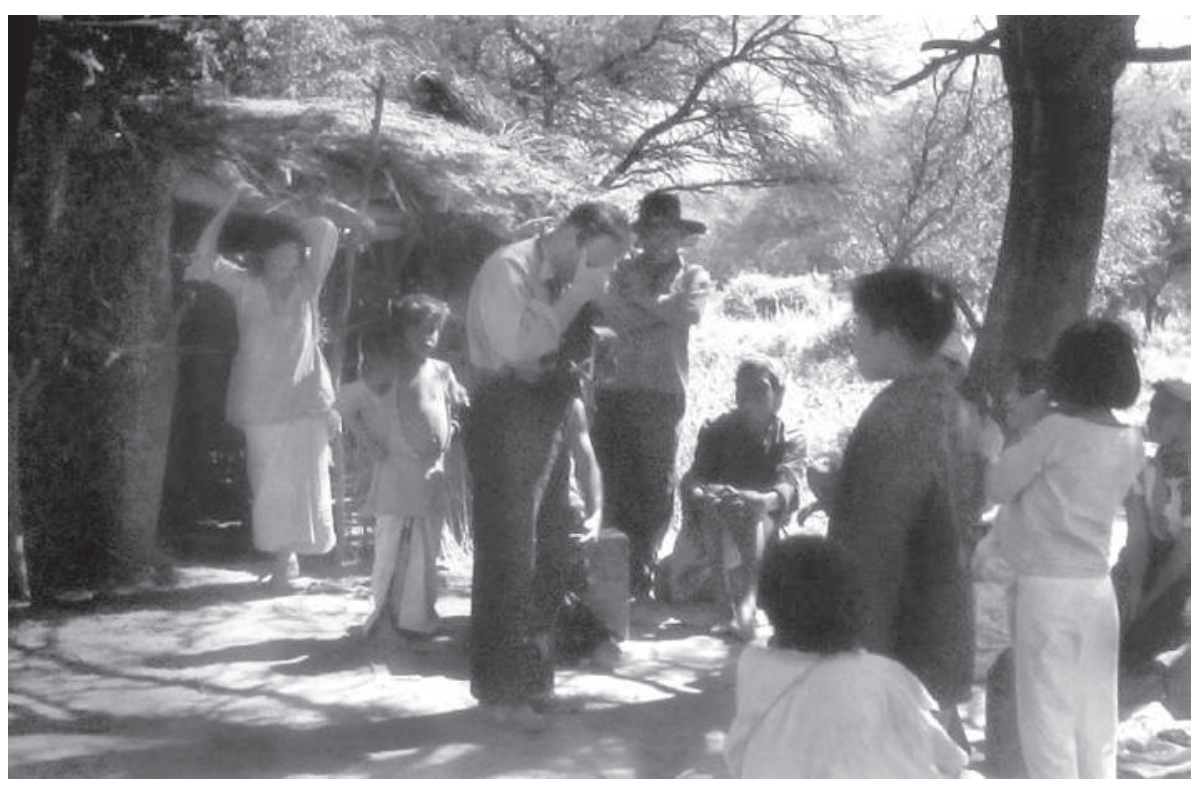

Fig. 2 - « A. M. et Indiens Pilagá », photo par Guy Métraux, 1939

(Guy Métraux C musée du quai Branly - Jacques Chirac).

1933, écrite à Misión E1 Toba, Métraux raconte en détail le départ de Misión Chaqueña, le passage par Misión San Andrés (Wichí), par Fortín Descanso et finalement l'arrivée à la mission ${ }^{22}$. Les impondérables n'en demeurent pas moins une constante:

Tout allait bien jusqu'à l'arrivée là-bas [Fortín Descanso], mais quand est venu pour moi le moment de revenir, c'est là qu'ont commencé les ennuis : impossible de trouver le moindre cheval. Tous seraient morts de la rage et je dus tant bien que mal parcourir seize lieues à travers bois en portant mes provisions comme rien moins qu'une mule de bât. Par le plus grand des hasards, j'ai pu obtenir un vieux canasson et réussi à me traîner jusqu'à la mission. Tout cela dura une semaine et me voici à présent à moitié boîteux et bien maigre. Pour comble de malchance, j'ai dû voyager un des jours les plus chauds de l'année. Voilà ce qu'il m'arrive toujours à force de voyager chichement. Quand est-ce que je vais enfin pouvoir monter une expédition en bonne et due forme ${ }^{23}$ !

22. Voir la carte en annexe qui mentionne les principaux lieux visités par Métraux en 1933. Avec les Chorote et les Nivaclé, les Wichí (famille linguistique mataco-mataguayo) apparaissent également dans les sources sous diverses désignations, telles « Mataco », "Vejoz », " Guisnay », « Noctene », etc. Il faut préciser que Misión San Andrés est aussi connue sous le nom de « Selva [forêt] San Andrés », de même que Misión Chaqueña est souvent appelée « Misión El Algarrobal » ou « Misión Chaqueña El Algarrobal ».

23. Lettre à Schreiter du 3 octobre 1933, citée in Amenta 2008, p. 222-223. 
Comme lors de sa première expédition, il se trouve vite contraint d'envoyer un télégramme à Schreiter pour qu'il lui fasse virer son salaire, faute duquel il ne pourrait entreprendre le voyage de retour. Et il rédige un autre rapport que publient les anglicans de Misión El Toba: «En tant que délégué de la 'Comisión de Indios', j'ai récemment visité chacun de nos établissements argentins et suis resté une paire de semaines à Misión El Toba, où je me suis renseigné sur la possibilité de fonder un territoire réservé pour nos Indiens ${ }^{24}$. » Comme le précédent, cet écrit se limite à deux pages et décrit le travail des missionnaires anglicans dans le Chaco argentin. Ces deux textes seront repris par Métraux pour la rédaction d'articles plus formels et plus conséquents pour le $J S A$ et Anthropos ${ }^{25}$.

\section{La mission}

Le 29 octobre 1930, les missionnaires anglais Alec Sanderson, Alfred Leake et Severiano (un Indien wichí de Misión Chaqueña) arrivent au lieu-dit Sombrero Negro pour y installer une réduction que les Indiens leur réclamaient depuis longtemps. La mission est baptisée « El Toba ${ }^{26}$. Cela faisait plusieurs années que, lorsqu'ils rencontraient des religieux à l'occasion de leurs migrations vers les raffineries de sucre des frères Leach dans la région de Salta, les Toba se plaignaient de l'absence d'une mission comparable à celle des Wichí (Misión Chaqueña). Leur principal argument était le besoin qu'ils éprouvaient de profiter de la présence des anglicans pour se protéger des intrusions des criollos (les créoles) et des militaires argentins ${ }^{27}$. Aussi les récits oraux contemporains insistent-ils régulièrement sur le rôle protecteur des missionnaires face aux abus: «Les missionnaires s'occupaient des gens, quand les soldats venaient, ils les défendaient, disant "je vais leur apprendre à vivre [...] leur enseigner à être des gens bien" 28 ». Les écrits des anglicans sont éloquents à cet égard, ainsi qu'il ressort clairement du journal du religieux en charge de la mission Chaqueña:

$1^{\text {er }}$ novembre 1928: le cinquième convoi de Toba est arrivé hier de Sombrero Negro, avide de nouvelles de nos « grands chefs » en Angleterre.

24. Métraux 1934, p. 15. Les religieux signalent qu'en octobre de cette même année, Métraux aurait sollicité de l'université de Tucumán une mise en disponibilité afin d'accompagner la Comisión de Reducciones de Indios, qui était alors dirigée par Juan A. Domínguez (Perilli de Colombres 2006, p. 151). Comme on le sait, cela ne put jamais se faire et Métraux partit à Honolulu pour occuper un poste au Bishop Museum.

25. Respectivement, Métraux 1933b et 1937.

26. Sur l'origine de Misión El Toba du point de vue missionnaire, voir Leake 1967; Lunt 2011, p. 40 et suiv.

27. Selon Gordillo 1999a, p. 121.

28. Entretien, communauté toba « Vaca Perdida », 2006. 
9 novembre 1928: des chefs toba arrivent de Sombrero Negro, espérant une fois encore les nouvelles apportées par le courrier (le courrier est arrivé sans nouvelle pour eux; ils sont repartis déçus).

21 novembre 1928: 11 chefs toba et environ 30 de leurs hommes qui vivent sur la rive paraguayenne du Pilcomayo veulent savoir si eux aussi peuvent envoyer leurs enfants à l'école.

8 décembre 1928: des Toba de Sombrero Negro sont arrivés aujourd'hui, confiants dans notre désir de les aider; ils se sont entretenus avec les autorités locales à propos du site pour l'école. Ils nous ont informés que tout serait décidé quand on leur aurait donné une lettre précisant que nous sommes bien d'accord pour nous occuper de l'école, ce qui nous est évidemment impossible.

11 décembre 1928: quelques Toba de Buena Vista arrivent pour nous assurer qu'ils seraient d'accord pour que l'école soit établie sur le site de Sombrero Negro.

7 janvier 1929: des Toba de Fortín Chasis, ayant entendu que le révérend E. Panter était venu renforcer notre équipe et pensant que c'était le missionnaire des Toba qui était arrivé, sont venus pour savoir quels étaient nos plans. Ils sont repartis très déçus.

22 janvier 1929: des Toba sont arrivés dans l'après-midi. Chefs de Formosa jusqu'à Buena Vista, 120 au total. Ils avaient entendu dire que M. Panter était là et sont venus à cheval dans l'espoir de le convaincre (et nous aussi) qu'un d'eux aille jusqu'à Sombrero Negro choisir un emplacement pour la mission ${ }^{29}$.

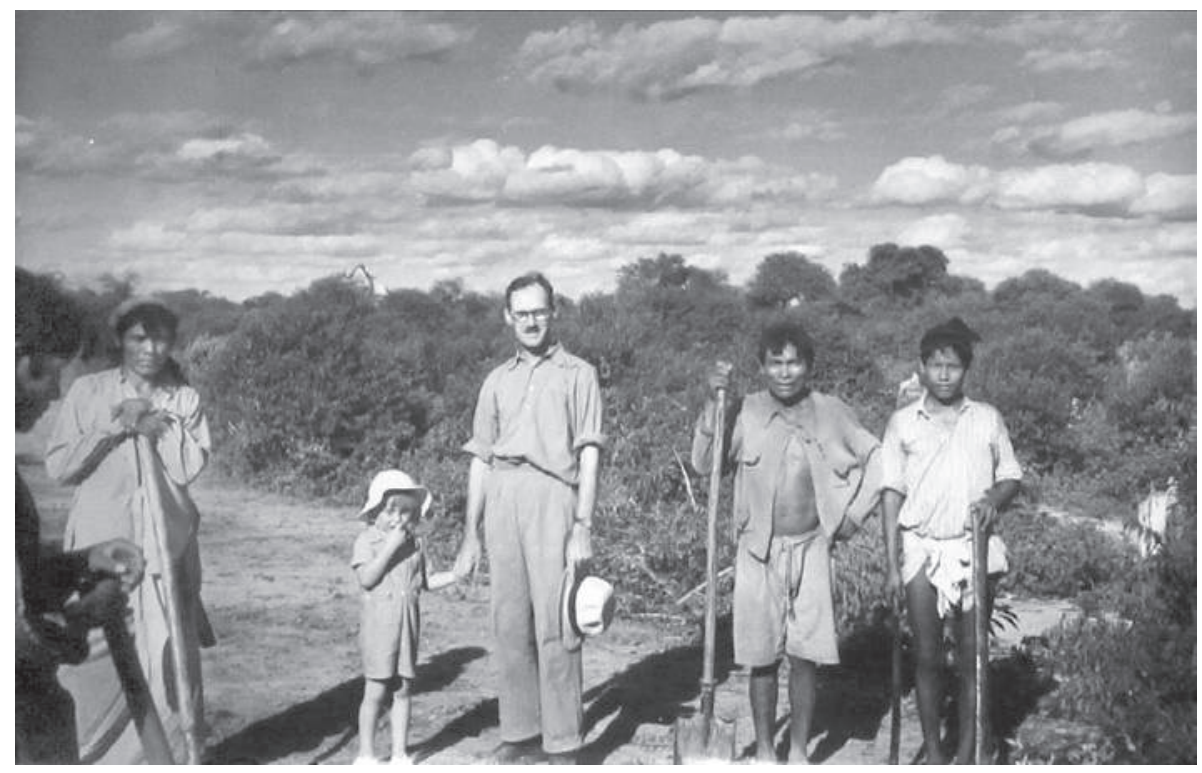

Fig. 3 - « Mister Leake, David et Indiens Toba », photo par Guy Métraux, 1939 (Guy Métraux (C) musée du quai Branly - Jacques Chirac).

29. Smith 1929, p. 43. 
Avant la guerre du Chaco, entre les années 1910 et 1930, plusieurs sources historiques rendent compte des affrontements belliqueux des Toba, Wichí et Pilagá avec l'armée. Dans ce contexte incertain, divers groupes ethniques contractent une alliance au cours du premier quart de siècle pour opposer une ultime résistance à l'intrusion colonisatrice : ainsi, perdure encore dans la mémoire des Toba l'affrontement de mars 1917 contre 50 soldats et 25 éleveurs où, pour étrange que cela puisse paraître, ce sont eux qui proclament avoir emporté la victoire bien que les sources démontrent le contraire. Quoi qu'il en soit, au cours des années suivantes - devant un bien sombre panorama marqué par des affrontements avec d'autres indigènes, des colons et aussi des militaires argentins, paraguayens et boliviens -, la protection des missionnaires pouvait apparaître comme la solution idéale :

En décembre 1928, il y eut une sérieuse confrontation entre les troupes boliviennes et paraguayennes, entraînant la rupture des relations diplomatiques et la guerre ne fut évitée que par l'intervention de la Ligue des Nations [...]. Que les hostilités se poursuivent ou non [...] le triste fait demeure que le travail des missionnaires de l'église dans cette région rencontre des obstacles et que les différentes tribus d'indiens du district sont considérablement énervées et persécutées ${ }^{30}$.

Métraux arrive à Misión El Toba alors que l'établissement a moins de trois ans d'existence. Il est dirigé par Alfred Leake, sa sœur Olive et John Arnott, avec lequel il nouera rapidement une relation d'amical respect qui plus tard transparaîtra dans son journal de voyage de 1939. C'est justement Arnott qui le présente à Kédok, avec qui il commence à approfondir l'étude de la culture toba. Métraux se défie des religieux et craint qu'ils « exercent une certaine pression sur les Indiens, ce qui entraverait inutilement leur liberté ${ }^{31} »$. Cependant, levées ces premières réticences, il en arrive à approuver le travail des missionnaires dans plusieurs secteurs de la vie communautaire: « Je commencerai par dire que c'est dans les missions anglaises que se trouvent les seuls Indiens heureux que j'ai jamais connus - et au cours de ces cinq dernières années de voyages et d'explorations j'ai connu un grand nombre d'Indiens ${ }^{32}$. »

L'opposition entre les agendas divergents des ethnologues et des missionnaires étant un des lieux communs de la tradition anthropologique, on peut s'étonner du regard chaleureux et des propos élogieux que Métraux tient à propos du

30. Chaqueño 1930, p. 33-34. Sur le travail des anglicans dans le Chaco en général, voir Torres Fernández (2006) et Gómez (2010) ; et sur leurs relations plus spécifique avec les Toba, voir Gordillo (1999b, 2002); Mendoza (2002); Arenas (2003); Córdoba et Braunstein (2008); Gómez C. (2008, 2011); Gómez M. (2011). Sur d'autres entreprises d'évangélisation dans la région, voir Ceriani Cernadas (2009) et le recueil de travaux publié dans Córdoba, Bossert et Richard (2015).

31. Métraux 1933b, p. 205.

32. Métraux 1934, p. 15. 
travail des anglicans ${ }^{33}$. La première surprise est la joie avec laquelle il est accueilli par les Pilagá de Descanso; comme ils ne le connaissent pas, il ne voit pas quelle pourrait bien être la cause de tant d'enthousiasme. Le bruit court de son arrivée et en peu de jours les chefs des autres communautés arrivent pour rencontrer le «nouveau missionnaire ». Métraux se rend alors compte que les Pilagá l'ont pris pour un missionnaire anglais et ils se montrent fort déçus lorsqu'il lève l'équivoque. La seule idée qui lui vienne, pour calmer les esprits, est de leur proposer d'aller en personne jusqu'à la mission la plus proche et d'y intercéder auprès des religieux pour qu'ils accèdent à leur demande de fonder leur propre mission ${ }^{34}$.

Les Indiens m'avaient confondu avec un missionnaire anglais et toutes ces réceptions reflétaient la joie que leur causait la perspective de voir s'établir parmi eux un de ces hommes dont ils avaient entendu parler avec tant d'affection par leurs frères de race. Ils projetaient déjà de me construire une école où ils « chanteraient » tous les jours. Ils rêvaient d'ériger un bâtiment pour la mission et à tout bout de champ se réjouissaient de la tournure nouvelle qu'allait prendre leur existence. «À présent, nous avons quelqu'un pour s'occuper de nous, pour nous instruire, maintenant nous ne sommes plus seuls et abandonnés et c'est une nouvelle vie qui commence $\gg{ }^{35}$.

J'aurais cru impossible qu'un village entier puisse se conformer si unanimement à la possibilité d'un changement de vie aussi complet. Ce désir collectif est le meilleur témoignage en faveur de l'efficacité et l'utilité du travail évangélique missionnaire. Pendant que j'étais chez les Pilagá je n'ai pratiquement rien su des missions protestantes et de leur travail. Mais je m'en suis fait la meilleure opinion possible en constatant la nostalgie passionnée que les indigènes éprouvaient à leur égard ${ }^{36}$.

Métraux se demande ce qui explique que les indigènes veuillent changer leur mode d'existence de façon si radicale. Cherchent-ils uniquement la protection des missionnaires? Il estime que les indigènes du Chaco voient l'existence dans les missions comme un moyen d'échapper à " une vie misérable » et comprennent que l'aide des religieux va de pair avec une nouvelle morale qu'ils sont disposés à accepter ${ }^{37}$. Ayant pu observer les ravages causés par la variole, il sait également que les normes d'hygiène et l'attention médicale des missions constituent une aide inégalable pour contrecarrer les effets de l'épidémie et que

33. Métraux 1933b, 1933c, 1934.

34. D'autres versions de ce récit peuvent être trouvées dans Métraux 1933b, p. 205; 1933c, p. $79 ; 1937$, p. 172. Le voyage jusqu'à Descanso se fit en compagnie du cacique Méndez de Guaikurú. Non loin de là, à Laguna de los Pájaros, Misión Pilagá sera fondée peu après (fin 1935), sous la responsabilité d'Arnott en personne, mais elle ne durera guère et sera finalement abandonnée en 1939.

35. Métraux 1933b, p. 205.

36. Métraux 1933c, p. 80.

37. Ibid. 
sans elles, les indigènes auraient péri en nombre bien plus élevé. Il éprouve en revanche un sentiment totalement différent à l'égard des quelques militaires argentins qui, loin d'aider les indigènes, opèrent au contraire comme agents de propagation de la maladie. « Certains chefs de fortins argentins, loin d'enrayer le mal, mettaient dans leur abstention une malignité hypocrite et cruelle. La mort de ces êtres inoffensifs et dignes d'un meilleur sort était pour ces militaires "un cadeau de nouvel an" selon la forte expression d'un poblador [colon] ${ }^{38}$. »

Par ailleurs, Métraux applaudit la réussite de l'école missionnaire chez les Indiens, ainsi que les possibilités que la mission leur offre de trouver un terrain d'entente raisonnable avec la culture nationale par l'acquisition de connaissances de base, telles que la lecture, l'écriture, l'arithmétique, la géographie et l'histoire argentines. Paradoxalement, remarque-t-il, la voie trouvée pour combler le fossé entre les « indigènes sauvages » et les « citoyens argentins » est l'instruction transmise par des missionnaires anglais. De fait l'arrivée des missionnaires augmente et facilite l'incorporation des Toba dans le contexte national, ces derniers acceptant non seulement une évangélisation permanente, mais aussi, plus fondamentalement, l'assistance médicale, l'apprentissage systématique de l'espagnol et même quelques projets de développement susceptibles de les insérer dans l'économie régionale, tels que l'élevage bovin ou la vente d'artisanat. En outre les missionnaires consolident leur projet en décourageant pendant quatre ans d'affilée la migration saisonnière vers les raffineries de sucre, renforçant ainsi la dynamique de sédentarisation. Tout cela, auquel s'ajoutent les compétences linguistiques accrues des anglicans, contribue à améliorer la réception de leur œuvre par les Indiens :

Les missions anglaises ont accompli leur œuvre dans l'obscurité, sans le moindre appui officiel elles ont obtenu des résultats bien supérieurs à ceux des entreprises militaires ou commerciales qui prétendaient « civiliser » le Chaco [...]. Aujourd'hui, trois missionnaires, deux hommes et une femme, administrent 700 Indiens Toba tandis qu'à quelques lieues de distance 300 soldats armés de mitraillettes sont nécessaires pour assujettir un nombre équivalent d'indigènes. Les faits sont éloquents : le Paraguay, la Bolivie et l'Argentine doivent beaucoup à l'œuvre silencieuse mais efficace des missions anglaises. Je ne pense pas exagéré d'affirmer que dans le Chaco, en ce qui concerne les indigènes, il n'existe aucune entreprise plus bénéfique pour la nation argentine que celle de ces infatigables missionnaires ${ }^{39}$.

En effet, les écrits de Métraux de ces années-là contiennent le plus souvent des appréciations plus ou moins positives de l'action des anglicans parmi les Indiens du Chaco. Par moments Métraux est si profondément affecté par ce thème qu'il va jusqu'à caresser l'idée de se convertir lui-même; il s'agit d'un épisode peu connu, qu'il décrit pour ainsi dire en passant, au détour d'une

38. Métraux 1937, p. 378-379.

39. Métraux 1933b, p. 208-209. 
lettre: «Plus que ma mission scientifique, je me suis occupé d'une mission protestante que j'ai essayé de fonder sur les rives du Pilcomayo. C'est mû par un élan inexplicable, peut-être par mon obscur atavisme huguenot que je me suis lancé dans cette aventure où j'ai mis tout mon cœur et tout ce que je possède en fait de volonté et de ténacité. Un jour je vous raconterai mes déboires et la façon tragique dont je suis sorti de ce rêve ${ }^{40}$. »

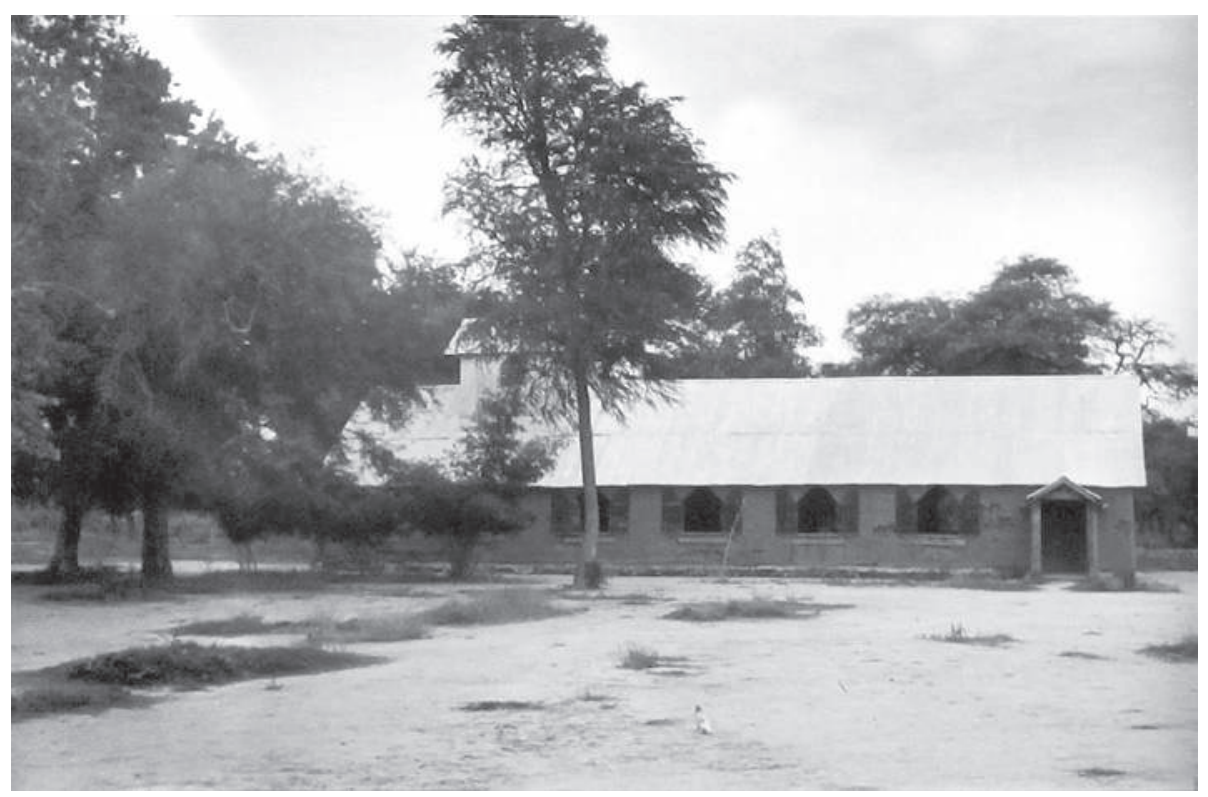

Fig. 4 - «L'église de Misión Chaqueña », photo par Guy Métraux, 1939 (Guy Métraux (C) musée du quai Branly - Jacques Chirac).

Cependant, après l'épiphanie, la réalité reprend le dessus et Métraux arrive à des conclusions plus équilibrées, marquées par une mélancolie notoire. $\mathrm{Si}$ d'un côté il apprécie les activités des missionnaires (les progrès de l'éducation, l'hygiène ou l'apprentissage professionnel), il sait également que la présence anglicane contredit par quelques aspects les objectifs de sa profession

40. Lettre du 27 avril 1933 à Yvonne Oddon (Yale). Christine Laurière (2005) observe également que « [...] le sort fait aux Indiens Pilagá du Pilcomayo le révolta profondément, au point qu'il crut un instant avoir été touché par le doigt de Dieu et qu'il pensa embrasser la vocation de missionnaire protestant afin de leur venir en aide ». Une analyse in extenso de 1'épiphanie religieuse de Métraux et de sa relation particulière avec le missionnaire écossais John Arnott a été proposée dans Córdoba (2015). 
d'ethnologue ${ }^{41}$. Si son objectif est de sauvegarder le souvenir de « ces petites civilisations ${ }^{42} »$, une partie de la responsabilité de leur disparition incombe aux missionnaires qui, de manière directe ou indirecte, déstructurent les activités traditionnelles que sont les luttes interethniques, les luttes entre femmes ou les fêtes arrosées de bière de caroube:

Une des choses qui nous surprirent le plus après avoir été si longtemps parmi les Toba - une des tribus les plus guerrières du Gran Chaco - était leur crainte sans raison apparente de provoquer le moindre malaise. Certaines coutumes dont ils pensaient qu'elles pourraient être contraires à nos enseignements cessèrent ou à tout le moins ne furent plus poursuivies que loin de nos yeux et de nos oreilles. Les danses natives cessèrent dans les environs de la mission, l'ancien jeu de hockey fut délaissé pendant plusieurs mois. Lors de la première saison sèche les fêtes de boisson furent bien tempérées ou cachées dans les bois loin de l'établissement [...]. Les premiers temps, ils nous demandaient de faire une quantité de choses des plus diverses: jeter un sort à un sorcier pour le faire mourir, récupérer des objets volés, chasser les éleveurs argentins qui occupaient le territoire toba ou encore faire pleuvoir ${ }^{43}$.

Une ambivalence similaire empreigne sa description - certes plus fragmentaire - des migrations saisonnières des indigènes jusqu'aux raffineries de sucre du nord-ouest de l'Argentine. Rappelons que lors des deux premiers voyages de Métraux les déplacements avaient été suspendus par les missionnaires et que de ce fait les Toba n'ont pas participé aux récoltes de 1931 à 1936. Lors du voyage de 1939, en revanche, Métraux rapporte dans son journal le joyeux chahut occasionné par l'arrivée des recruteurs ${ }^{44}$. D'un côté, les anglicans désapprouvent les mœurs peu chrétiennes qui prédominent dans les raffineries (jeu, boisson,

41. Ainsi, dans son récit d'une visite ultérieure à Misión El Toba en 1939, il consigne la discussion qu'il eut avec Alfred Leake: « Ce White et ce Leak [Leake], deux fanatiques, ont l'air de s'entendre. Je suis étonné du fanatisme de Leak qui dit ne compter pour rien l'œuvre sociale accomplie dans la mission et ne voir que la conversion des Indiens au Christ: faire leur salut, tel est son seul but. Il n'approuve une ethnographie que si elle est faite par un chrétien: les ethnographes lui apparaissent comme des suppôts du paganisme, etc. Je finis par lui dire combien j'ai été froissé par ses paroles et je lui tends la main. Le geste semble le toucher. Toute cette scène m'a excité et je peine à m'endormir» (Métraux 1978, p. 78). Ces propos tardifs de Métraux sur les anglicans doivent être compris dans leur contexte, en tant qu'extraits d'un journal intime clairement sujet aux fluctuations d'humeur de l'auteur.

42. « C'est, je crois, cette idée, réellement fondamentale, qui a inspiré ma carrière d'ethnographe: j'ai voulu conserver le souvenir, ou l'image, de ces petites civilisations. Elles vont mourir, sans aucun doute, elles meurent déjà : en trois, quatre ans, j'ai vu des peuplades, sinon disparaître physiquement, du moins perdre toutes leurs traditions, se fondre dans une masse amorphe. Néanmoins, il est utile, profitable, de conserver une image aussi précise que possible de leur type de vie. Je dis: aussi précise que possible, parce qu'en ce domaine il s'agit de faire œuvre scientifique » (cité in Bing 1964, p. 23).

43. Leake 1933a, p. 67; voir Leake 1933b.

44. Métraux 1978, p. 73. 
bagarres, etc.); mais d'un autre côté, ils ne sont pas totalement opposés aux migrations de travail, en partie en raison de leurs liens étroits avec certains des propriétaires des raffineries, mais aussi en vertu d'une affinité qu'on pourrait qualifier de «weberienne » entre l'éthique protestante et l'insertion dans le marché du travail, et aussi, plus fondamentalement, à cause de l'attitude favorable des Indiens eux-mêmes.

\section{La guerre}

En janvier 1933, en pleine guerre du Chaco, Métraux publie dans un journal de Buenos Aires, La Prensa, un article intitulé: « La guerre primitive dans le Chaco ».

Par son caractère, par son environnement et j'irais jusqu'à dire pour des raisons ethniques, les hostilités qui se déroulent actuellement dans le Chaco évoquent le souvenir d'autres conflits qui eurent pour théâtre ces mêmes fourrés et comme protagonistes ces indigènes dont le sang court encore dans les veines des soldats mobilisés dans les armées belligérantes de la Bolivie et du Paraguay ${ }^{45}$.

Il ne s'agit pas seulement de souvenirs nostalgiques. Au cours de l'affrontement qui les oppose entre 1932 et 1935, les armées de la Bolivie et du Paraguay annexent les groupes indigènes du Chaco, les arment et, parfois, ravivent d'anciennes inimitiés:

Au retour et par le même chemin, si tant est qu'on puisse appeler ça un chemin, qui mène à Salto Palmar, je m'étonne de l'absence d'Indiens dans les champs, alors qu'ils y sont si nombreux en Argentine. On m'informe qu'ils sont regroupés dans les fortins. La race indigène dans cette zone est celle des Macá, guerriers féroces et implacables qui rendent d'inestimables services comme pisteurs, ou pour surprendre les sentinelles et les éclaireurs. Les Boliviens ont également recours aux auxiliaires indigènes, en l'occurrence les non moins valeureux Chunupi $^{46}$.

Pour diverses raisons historiques, géographiques, culturelles ou liées à la stratégie militaire, certains groupes se voient plus impliqués que d'autres, chacun d'entre eux étant dès lors diversement affecté par le conflit ${ }^{47}$. Ainsi, par exemple, pour les Wichí-Guisnay qui habitent au nord du bras principal du Pilcomayo, la guerre entraîne le retrait du territoire paraguayen et la traversée définitive du fleuve du côté argentin. Pour les Pilagá, installés en aval du côté argentin de la

45. Métraux 1933a. Il semblerait qu'à leur retour du terrain, il était assez habituel pour les ethnologues français d'écrire simultanément des textes scientifiques et d'autres destinés au grand public (Debaene 2014). Il est à cet égard notable que Métraux, pour sa part, écrivit en même temps des travaux pour le $J S A$, un article pour La Prensa et le texte apologétique qu'il donna aux missionnaires, en plus de sa correspondance personnelle et professionnelle.

46. Da Rocha 1937, p. 94.

47. Voir les différentes études compilées dans Richard (éd.) 2008, 2011 et Capdevila et al. 2010. 
frontière internationale, la guerre a un impact nettement moindre et, en raison des alliances contractées avec l'armée nationale les années précédentes lors de la délimitation des frontières, ils voient simplement se renforcer leur statut « d'indigènes argentins » par contraste avec les Nivaclé ou les Maká. Pour les habitants de Misión El Toba, en revanche, le conflit implique la restriction de leurs incursions de l'autre côté du Pilcomayo, de sorte que « la frontière » cesse d'être considérée comme partie intégrante de leur territoire de chasse, de pêche et de cueillette ${ }^{48}$.

Combinant les souvenirs de la mémoire orale toba avec les témoignages d'ethnographes, de militaires et de missionnaires de l'époque, on peut reconstituer le contexte dans lequel se forma l'expérience de Métraux en 1933. Quand on interroge les Toba sur la guerre, une des premières questions qui surgit est l'arrivée des déserteurs boliviens « coya $^{49}$ » qui se cachent parmi eux pour échapper à l'armée paraguayenne:

Quand il y eut la guerre contre la Bolivie et le Paraguay, de nombreux Coya sont venus penauds par ici. Aujourd'hui, il y a des Coya à Juárez qui tiennent des commerces, mais ceux-là sont nouveaux [...]. De nombreux criollos ont traversé pour venir de ce côté-ci de la frontière parce que le gouvernement du Paraguay les faisait payer pour faire paître le bétail, alors qu'ici ils ne payaient rien [...]. Le père de Hueso (l'actuel agent sanitaire criollo) était un miliaire coya, qui est venu fuyant les Paraguayens. Il s'est marié avec une criolla et Hueso (le fils) s'est marié avec une petite Toba [...]. Lorsqu'arrivent les enfants de Hueso, ils disent « voici venir les Coya » [...]. Il se souvient que son papa lui racontait la guerre entre la Bolivie et le Paraguay. Beaucoup de prisonniers boliviens échappés sont venus par ici. En ce temps-là, l'Argentine ouvrait grandes ses portes. On entendait les tirs [...]. Les prisonniers coya traversaient la frontière, s'enfuyaient jusqu'ici [...]. Les Coya venaient pour se sauver, pour se cacher ici avec les aborigènes, pour se sauver ${ }^{50}$.

De nombreux soldats boliviens, déserteurs ou fugitifs des camps de prisonniers, ont traversé le fleuve pour se réfugier dans les communautés indiennes des berges. Les Toba en ont gardé le souvenir, confirmé par les nouvelles du front conservées dans les archives militaires paraguayennes: "Communiqué $\mathrm{n}^{\circ} 58$ : le consul national de Formosa (RA) communique au ministère qu'au lieu-dit Sombrero Negro est apparu un contingent de déserteurs boliviens menés par un capitaine [...] Asunción, novembre $1932^{51} »$. Les Boliviens ne sont pas tous

48. Pour une analyse détaillée de l'impact de la guerre sur les Indiens du moyen Pilcomayo, voir Córdoba et Braunstein 2008.

49. « Coya » provient de Colla, originellement le nom d'Indiens du lac Titicaca et, par extension, terme appliqué aux Indiens boliviens des Andes en général. Ici, « Coya » est le nom générique que les Toba donnent à tous les Boliviens.

50. Entretien, communauté toba « Vaca Perdida », 2006.

51. Comando en Jefe de las FFAA de la Nación 1950, p. 50. L'auteur remercie Hugo Beck de l'avoir aidée à obtenir cette source. 
bien reçus; on a même rapporté que des déserteurs se seraient fait tirer dessus en voulant traverser à la nage: « Les hommes allaient pêcher dans le fleuve et veillaient à ce que personne ne passe. Certains n'hésitaient pas à tuer ceux qui essayaient de passer ${ }^{52}$.» Quoi qu'il en soit, en dépit de ces escarmouches, une quantité appréciable de migrants s'est installée à proximité, voire à l'intérieur des communautés indiennes, ou dans la localité criolla d'Ingeniero Juárez, où l'on se consacre principalement au commerce. Se voit ainsi consolidée l'incorporation active des « Coya » au réseau de relations sociales tant au niveau local, dans la vie quotidienne indienne, que dans le cadre de l'économie régionale ${ }^{53}$.

En plus de l'apparition de nouveaux acteurs sociaux, la mémoire toba recompose un tableau lourd d'incertitudes, marqué par les affrontements, la restructuration des territoires indiens et la transformation subséquente des relations interethniques:

Au cours de l'été 1933, les officiers et soldats boliviens ont assassiné le cacique Nayocolik et trois de ses enfants qui chassaient pacifiquement des loutres sur la rive gauche du Pilcomayo. Quelques Pilagá ont traversé le fleuve et réussi à tuer une sentinelle bolivienne ${ }^{54}$.

Les premiers jours du trimestre ont été assombris par la mort de Yanagadi et l'incertitude concernant la réaction des Paraguayens suite à ce triste et malencontreux événement. Les gens se rendaient au fleuve la peur au ventre et on a même vu des hommes aller pêcher avec un filet dans une main et une arme dans l'autre. Les Indiens avaient évidemment leur part de responsabilité, mais il est bien malheureux que les Paraguayens aient pris des mesures si drastiques et, comme toujours, qu'un innocent paye de sa vie une offense mineure commise par ceux pour qui l'Évangile signifie bien peu [...]. Il a été capturé avec un compagnon tandis qu'il se préparait pour une nuit de pêche sur la rive opposée du fleuve, où il n'y avait aucune restriction sur la pêche ${ }^{55}$.

52. Entretien, communauté toba «Vaca Perdida », 2006. Voir aussi les témoignages recueillis dans De la Cruz (1995). Dans la liste des toponymes toba figure coyalée lcoló, défini comme un des havres du fleuve dont le nom pourrait se traduire par « cul du Coya », « le Coya a chié », « le cadavre s'est enflé et son lcoló, l'intestin du dedans, lui est sorti par le cul », etc. Selon De la Cruz (1995, p. 85): « [Du temps de la guerre entre les Boliviens et les Paraguayens] il y avait des Boliviens [qui] passaient en Argentine; mais les Toba étaient mauvais; s'ils ne les connaissaient pas, [ils les tuaient] et les jetaient à l'eau. Les Boliviens voulaient venir de ce côté-ci, mais nos ancêtres les tuaient et les jetaient à l'eau. Ce lieu étant un havre, l'eau ne s'écoule pas vers l'aval mais tourne plutôt en boucle, et du coup le corps y reste, flotte et enfle jusqu'à ce que les choses du dedans lui ressortent par le cul ». Pour une comparaison avec le cas nivaclé, voir Capdevila et al. 2010.

53. De fait, à l'issue du conflit, on vit augmenter le flux des travailleurs boliviens vers ces mêmes plantations de canne à sucre où se rendaient les Wichí, Toba et Pilagá du Pilcomayo (Gordillo 1999a, p. 154).

54. Métraux 1937, p. 397.

55. Leake 1937, p. 127. 
Pour de nombreux Toba, la guerre entre la Bolivie et le Paraguay se réduit cependant à rien de plus qu'une série d'escarmouches occasionnelles avec les soldats lorsqu'ils allaient pêcher ou puiser de l'eau à la rivière, affrontements au cours desquels quelque parent pouvait être blessé : « Personne de par ici ne s’est battu dans cette guerre. Les gens, les Toba regardaient, c'est tout, ils venaient, voyaient, rien de plus... Lors des combats, ils ne faisaient que regarder ${ }^{56} \ldots$ » Pour ces témoins, l'impact de la guerre paraît n'avoir été que fragmentaire et indirect ${ }^{57}$. Pour mieux comprendre le degré d'incidence de la guerre sur la vie quotidienne, il faut donc récapituler brièvement l'histoire du groupe et évaluer le poids relatif du conflit en relation avec les autres événements.

Pour la grande majorité des Toba, en effet, la guerre est loin d'être « le » grand événement marquant de l'histoire de ces années-là. Le conflit qui se déroule de l'autre côté du fleuve n'est guère qu'un incident de plus, qu'il faut mettre en relation avec d'autres faits plus ou moins contemporains. Une première source de contact intensif avec la société occidentale s'était déjà mise en place par le biais de l'insertion dans l'économie régionale, tant pour la récolte de canne à sucre que par le biais du travail salarié pour la construction des voies de chemin de fer entre Salta et Formosa. Une seconde source apparut entre 1910 et 1930, lorsque les Toba durent se confronter à l'arrivée massive de colons criollos qui commençaient à peupler la zone de manière systématique et qui rémunéraient souvent les chefs avec des cadeaux (objets métalliques, bétail, vêtements), obtenant ainsi l'autorisation de faire paître leurs animaux sur les terres communautaires. Cette pression de la population criolla, indissociable de l'avancée de l'élevage, suscita d'assez nombreux affrontements entre colons et Indiens.

Voilà pourquoi Métraux prévient d'emblée que, pour les Toba, c'est l'arrivée des missionnaires anglicans qui constitue le fait historique marquant de cette époque. La tradition orale se fonde sur une chronologie qui oppose le temps « des anciens », avant la mission, aux temps « nouveaux », qui lui sont contemporains. Cette fracture historiographique permet d'opposer distinctement les valeurs négatives du passé (la violence ritualisée d'antan, la chasse aux scalps, les fêtes de boisson, l'ignorance de la technologie et des enseignements religieux) et celles, plus positives, typiquement attribuées à la modernité (la technologie, l'hygiène, la salubrité, le « message du Christ » et la « civilisation »). Pareille torsion narrative n'est cependant pas exempte de tensions significatives, comme

56. Entretien, communauté toba « Vaca Perdida », 2006.

57. « Au début de 1934, la guerre du Chaco entre la Bolivie et le Paraguay (déclenchée en 1932) impliqua directement le territoire jouxtant Sombrero Negro et Misión El Toba, lorsque les Paraguayens poussèrent une offensive vers l'ouest suivant le Pilcomayo. Les fortins boliviens du fleuve se mirent à tomber l'un après l'autre. Début janvier 1934, les Paraguayens prirent Fortín Esteros (en face de Nuevo Pilcomayo) et quelques jours plus tard eut lieu un affrontement important non loin de Magariños, le fort situé juste en face de El Toba. Mais même ainsi, ces batailles n'ont pas affecté directement les Toba » (Gordillo 1999a, p. 127). 
lorsqu'on admet que du temps des anciens on ne connaissait ni la faim, ni les maladies, ni la pauvreté, et que l'alimentation actuelle est nettement moins diversifiée que la traditionnelle.

En plus de la sédentarisation, de l'assainissement et de l'action évangélisatrice, l'impact majeur des missionnaires aura probablement été l'instauration d'une Pax Anglicana ${ }^{58}$. Les religieux désamorcent de manière explicite et systématique les affrontements interethniques traditionnels des Indiens du Chaco: «Les anciens d'avant se battaient contre les Chulupí, les Wichí. Jusqu'à ce qu'arrive Alfredo (Leake) qui a dit que nous sommes tous frères; et ça ne lui plaît pas que les gens se battent ${ }^{59}$. \ Le discours des missionnaires va dans le même sens :

À part ce travail régulier dans divers secteurs, la mission a aussi dû agir comme médiatrice entre les Indiens et les Blancs et aussi dans la résolution des disputes des Indiens entre eux ${ }^{60}$.

L'état de guerre qui règne sur l'autre rive du Chaco boréal a grandement perturbé les Indiens riverains et beaucoup d'entre eux ont dû se disperser dans diverses directions. La tribu connue sous le nom de Chunupi [Nivaclé ou Chulupí], dont certaines familles vivent depuis des générations sur la rive argentine du Pilcomayo, n’ont pas connu de répit. Ses membres ont traversé le fleuve pour visiter aussi bien la station à Selva San Andrés que Misión El Toba. En ce dernier lieu, quelques Chunupi se sont établis pour l'instant. Cela est hautement significatif. Les Chunupi et les Toba sont ennemis jurés. Les premiers jours sur le Pilcomayo, les premiers rendaient occasionnellement visite aux Mataco [Wichí] qui vivaient à la mission, mais ils évitaient toujours les Toba. À une occasion, tandis qu'un groupe de ces derniers était employé par quelque colon blanc, les Toba sont arrivés et les travailleurs ont tout lâché pour s'enfuir vers leurs maisons. Mais ici, ils vivent en toute tranquillité avec leurs anciens ennemis et vont même jouer et chasser ensemble ${ }^{61}$.

Au cours des anciennes guerres intertribales, la prise de captifs et l'assimilation subséquente des enfants à la bande des vainqueurs entraînait un métissage entre des groupes qui s'affrontaient sur le plan militaire mais n'en pratiquaient pas moins l'alliance matrimoniale. Les Toba affrontaient d'un côté les Nivaclé ou Chulupí, et de l'autre différents groupes de Wichí. La guerre n’était pas seulement une manière d'entrer en contact avec les ethnies voisines mais aussi une façon de donner substance à divers aspects de la vie sociale et symbolique de par ses liens avec le chamanisme, la conception de la personne, les activités

58. Par exemple: « Beaucoup de gens estiment qu'une des “nouvelles” valeurs apprises des missionnaires a été de "vivre en paix" et "aimer ses frères"... » (cité in Gordillo 2002, p. 184).

59. Entretien, communauté toba « Vaca Perdida », 2006.

60. Leake 1933a, p. 69.

61. Leake 1933b, p. 116. 
économiques et même avec la morphologie saisonnière des campements ${ }^{62}$. Bien que certains informateurs estiment que les disputes découlaient essentiellement de problèmes liés à la gestion du fleuve, et en particulier des espaces de pêche, d'autres intérêts n'en étaient pas moins en jeu: l'acquisition de biens matériels et de bétail, la prise de captifs, la vengeance ou la rétribution des hostilités.

Dans le Chaco, la population indigène étant rare et le territoire immense, les litiges de frontière ne devraient guère tirer à conséquence, mais là-bas, on ne se dispute pas tant pour la terre que pour l'eau. Les puits d'eau et les lagunes qui, pendant la saison des pluies, permettent à des petits groupes d'hommes de vivre à l'intérieur, s'assèchent pendant les mois d'hiver et les indigènes sont obligés de se concentrer aux alentours des ruisseaux où l'eau coule encore ou à s'installer sur les rives du Pilcomayo [...]. Chez les Indiens du Pilcomayo la paix ne se négocie pas entre tribus, mais entre individus. On se met d'accord sur le montant du prix du sang à payer aux parents des victimes de la dispute en moutons, chevaux et autres biens [...]. Au cours d'une de leurs guerres, les Ashluslay [Nivaclé ou Chulupí] ont tenté de racheter leurs enfants prisonniers des Toba en leur donnant des chevaux ${ }^{63}$.

Avec l'arrivée des missionnaires, les disputes interethniques se restreignent drastiquement, ou plutôt se mettent à emprunter les voies ad hoc du mépris sournois ou de la réduction des échanges matrimoniaux. Les affrontements ne cessent pas pour autant et tout indique que les Toba profitent de la guerre entre la Bolivie et le Paraguay pour se procurer des armes qui leur serviront ensuite contre leurs ennemis traditionnels. En effet, bon nombre d'entre eux traverse « la frontière » pour récupérer ce qui a été disséminé sur le champ de bataille: «On trouve encore dans le Chaco des restes de matériel belliqueux, bien que les Indiens les recherchent avec le plus grand intérêt », prévient un militaire argentin ${ }^{64}$. Le missionnaire responsable de Misión Pilagá rapporte la même chose :

Si on les écoutait, les Indiens aimeraient bien retourner sur les sites de leurs anciens villages mais cela ne leur est pas permis parce que ces derniers se trouvent en zone militaire. Une autre plainte est que les Pilagá sont armés de fusils et associés avec les Chunupí, qui passent pour très sauvages. Certains des jeunes gens d'ici ont des fusils qu'ils ont achetés aux Chunupí, qui les ont pour leur part récupérés sur les anciens champs de bataille entre la Bolivie et le Paraguay. On essaye de les convaincre d'abandonner ces armes illégales mais c'est bien difficile [...].

62. Parmi les éléments à forte valeur symbolique, se distingue tout particulièrement la chasse aux scalps (cuirs chevelus des ennemis tués au combat), lesquels, une fois séchés, servaient de trophées et de récipients pour la boisson durant les fêtes de célébration de la victoire (Métraux 1937, p. 378-379; voir Nordenskiöld 2002 [1912]; Clastres 1987; Tomasini 1990; Sterpin 1993; Arenas 2003, p. 67-75).

63. Métraux 1933a.

64. Vaca 1938, p. 140. 
Une autre raison pour laquelle ils ne veulent pas s'en défaire est la peur, une fois désarmés, de devenir des proies faciles pour les soldats ${ }^{65}$.

Les anglicans sont parfaitement conscients que, la plupart du temps, les armes que les indigènes utilisent pour se défendre de l'armée ou des colons sont également utilisées pour attiser de vieilles inimitiés:

Depuis que nous vivons avec eux, les Toba ont cessé de prendre activement part à des guerres ouvertes, bien que tout nous rappelle sans cesse que les tueries n'ont pas cessé. Une petite fille, lors d'une promenade un dimanche après-midi, nous commenta par exemple, alors qu'on traversait un bosquet: " c'est ici qu'ils ont tiré sur le vieux Mataco qui a été tué l'autre jour ». Ou quand un homme s'est soudain orné d'une coiffe de « meurtrier » et qu'un de ses parents est venu quelques jours plus tard essayer de nous vendre une magnifique paire d'éperons en argent. Ou lors de la guerre du Chaco, quand nous arrivaient des histoires de massacres de déserteurs boliviens. Si la guerre ouverte a cessé, on ne peut pas en dire autant de l'arrogance et de l'orgueil ${ }^{66}$.

Une fois terminée la guerre entre la Bolivie et le Paraguay, les affrontements ne cessent pas pour autant entre les troupes paraguayennes et les Toba, qui continuent de traverser le fleuve pour aller chasser ou récupérer des armes, même s'ils savent qu'ils risquent de tomber sur les militaires surveillant la frontière. Ce n'est que plusieurs années après la fin du conflit que les missionnaires constatent l'arrêt définitif des hostilités entre Paraguayens et Toba, et même la célébration d'une sorte de pacte entre chacune des bandes qui règle la traversée du fleuve:

Nous sommes heureux de rapporter que ce dernier trimestre a été totalement dépourvu d'affrontements entre les Toba et les soldats paraguayens. Les deux bandes avaient mutuellement peur l'une de l'autre, mais une ou deux fois, quand elles se sont rencontrées, elles ont échangé des saluts. Il ne s'est produit aucun incident tandis que les Indiens pêchaient dans le fleuve, essentiellement parce que ces expéditions de pêche évitaient l'autre rive du Pilcomayo ${ }^{67}$.

Bien que Métraux n'ait jamais publié d'article spécifiquement consacré à la guerre, il a en revanche fréquemment écrit au sujet du conflit. Parfois de manière explicite: «nous sommes passés par Vilamontes [Villa Montes], quartier général de l'armée bolivienne. Je vous assure que le spectacle était

65. Tebboth 1937, p. 85.

66. Leake 1970, citée in Mendoza 2002, p. 82.

67. Tebboth 1938, p. 19. Toutefois, cette même année (1938), le missionnaire Arnott envoya un rapport privé au directoire de la SAMS à Londres où il relatait les affrontements qui eurent lieu à Misión Pilagá entre les militaires, les criollos et les indigènes. Selon le récit d'Arnott, une des préoccupations majeures des militaires est la quantité d'armes abandonnées durant la guerre du Chaco et que se sont appropriés les groupes toba, pilagá et chulupí (WUA, Jules Henry Papers, serie 9, box 10, folder 5, letters pilagá 8 y 9). 
bien bolivien: crasse, stupidité, méchanceté, inconfort. Il y avait pas mal de blessés, mais il semblerait qu'ici, on en fasse peu de cas. Les soldats, tous de purs Indiens, font peine à voir: mal vêtus, mal nourris, paraissant totalement abrutis $^{68} \gg$. D'autres fois, comme dans l'article déjà cité de La Prensa, il décrit la guerre de manière plus indirecte, proposant un certain type de continuité intuitive, sous-jacente, comme s'il s'agissait d'une référence collatérale, une toile de fond pittoresque mais non moins inquiétante pour autant:

Entretemps, j'étais resté sur la rive argentine du fleuve, vivant avec mes amis, les Indiens Toba. Les nuits claires, assis sur la berge du fleuve à parler des anciens temps, nous entendions souvent un bruit étrange et inquiétant dans ces solitudes: c'était le canon des forts boliviens. Certes, ce n'était pas le grondement continu et effrayant que nous écoutions il y a vingt ans [mois], mais le coup de tonnerre isolé d'un orage distant. Rarement dans mes voyages, je n'ai éprouvé sentiment d'angoisse plus pénible qu'à chacune de ces détonations qui dissipaient à jamais la calme vie primitive des plaines du $\mathrm{Chaco}^{69}$.

\section{Conclusion}

Lorsque Métraux rendit visite aux Toba et Pilagá du Pilcomayo, au cours de ses voyages de 1933, la guerre du Chaco sévissait de l'autre côté du fleuve, à quelques kilomètres à peine des villages et des missions qu'habitaient les Indiens. Les militaires argentins, au même moment, renforçaient avec zèle leurs lignes de fortins afin de protéger les frontières internationales qui les séparaient des nations belligérantes. Le jeune ethnographe percevait toutefois la guerre, au moins partiellement, comme un affrontement entre Indiens et, de manière plus importante, il allait jusqu'à suggérer quelques lignes d'interprétation plus ou moins implicites permettant d'y voir un ultime avatar des guerres interethniques régionales. Autrement dit, en identifiant le jeu des ruptures et des continuités, Métraux parvient à dépeindre la guerre à partir du point de vue et en tenant compte de l'expérience vécue des populations indiennes elles-mêmes. Son flair d'ethnographe lui permet de soupeser l'importance du conflit à l'aune des autres faits historiques contemporains tels que l'installation des missionnaires anglicans qui, non contents de démembrer les vieux cycles d'affrontements institutionnalisés entre les Indiens du Chaco, avaient aussi altéré, directement ou indirectement, plus encore que la guerre elle-même, leur rapport au territoire, leur mode de résidence, leurs pratiques économiques, leurs réseaux d'alliance

68. Lettre à Schreiter du 18 septembre 1933, citée in Amenta 2008, p. 220.

69. Métraux 2013 [1937], p. 431. On pourrait également évoquer le rôle de Métraux dans la guerre elle-même, par exemple la fameuse anecdote à laquelle se réfère Pierre Clastres: Métraux prévenant les Nivaclé de l'imminence d'une attaque des troupes frontalières argentines, leur sauvant ainsi la vie (Clastres 1994, p. 133). 
et de parenté ainsi que leurs relations interethniques au sens le plus ample du terme. Tantôt plus critique (par exemple lorsqu'il dépeint la décomposition des coutumes traditionnelles), tantôt plus apologétique (lorsqu'il décrit les progrès affectant les Indiens sur les plans de la santé, de l'économie ou de l'éducation), l'importance accordée à l'œuvre missionnaire se manifeste dans toute sa splendeur à l'heure de décrire les effets concrets de la Pax Anglicana sur la cartographie des relations interethniques à l'échelle régionale.

Pourrait-on dès lors dire que Métraux « oublie », « escamote » ou « ignore » la guerre du Chaco, lui niant toute valeur explicative? Il semblerait que non. À tort ou à raison, il a tenté de comprendre la guerre comme un affrontement entre Indiens. Il la dépeint depuis la perspective toba, à l'aune de la possibilité d'accéder au fleuve pour la pêche, d'obtenir des armes, de capturer des prisonniers, de prendre des scalps ou de renouer avec les cycles de vengeance réciproques qui liaient entre elles les différentes sociétés indiennes du Pilcomayo. Il n'envisageait pas la guerre, ou en tout cas pas seulement, comme un phénomène propre à l'histoire institutionnelle, avec un $\mathrm{H}$ majuscule; en bien ou en mal, il ne pouvait s'empêcher de l'interpréter comme un processus de changement social parmi tant d'autres. De fait, sa perception reflète strictement les critères de la conscience historique toba, pour laquelle l'événement historique marquant de l'époque, celui qui structurait leur expérience, était avant tout l'arrivée des missionnaires anglicans. Ce ne sont donc pas les « civilisations qui meurent ${ }^{70} »$, mais les guerres, supposées les définir, qui disparaissent - ce qui revient à soulever ni plus ni moins qu'une nouvelle énigme anthropologique. ${ }^{71} *$

* Manuscrit reçu en janvier 2016, accepté pour publication en juin 2016.

Remerciements - Nos remerciements aux archives Miguel Lillo du Centro Cultural Alberto Rougés de Tucumán et à celles de l'Église Anglicane à Buenos Aires (en particulier à David Leake, Robert Lunt et Cristobal Wallis); à Daniel Métraux, à Fernande Schulmann-Métraux ( $\dagger$ ) et Guy Métraux pour l'autorisation de reproduire la correspondance et les photographies de Métraux; à Sophie Assal, Miranda Rectenwald, José Braunstein, Federico Bossert, Isabelle Combès, Philippe Erikson, Cecilia Gómez, Christine Laurière, Rodrigo Montani, María M. Sampietro, Marta Tartusi et Diego Villar pour les échanges d'idées et de matériaux.

70. Métraux cité in Bing 1964, p. 23.

71. Traduit de l'espagnol par Philippe Erikson. 


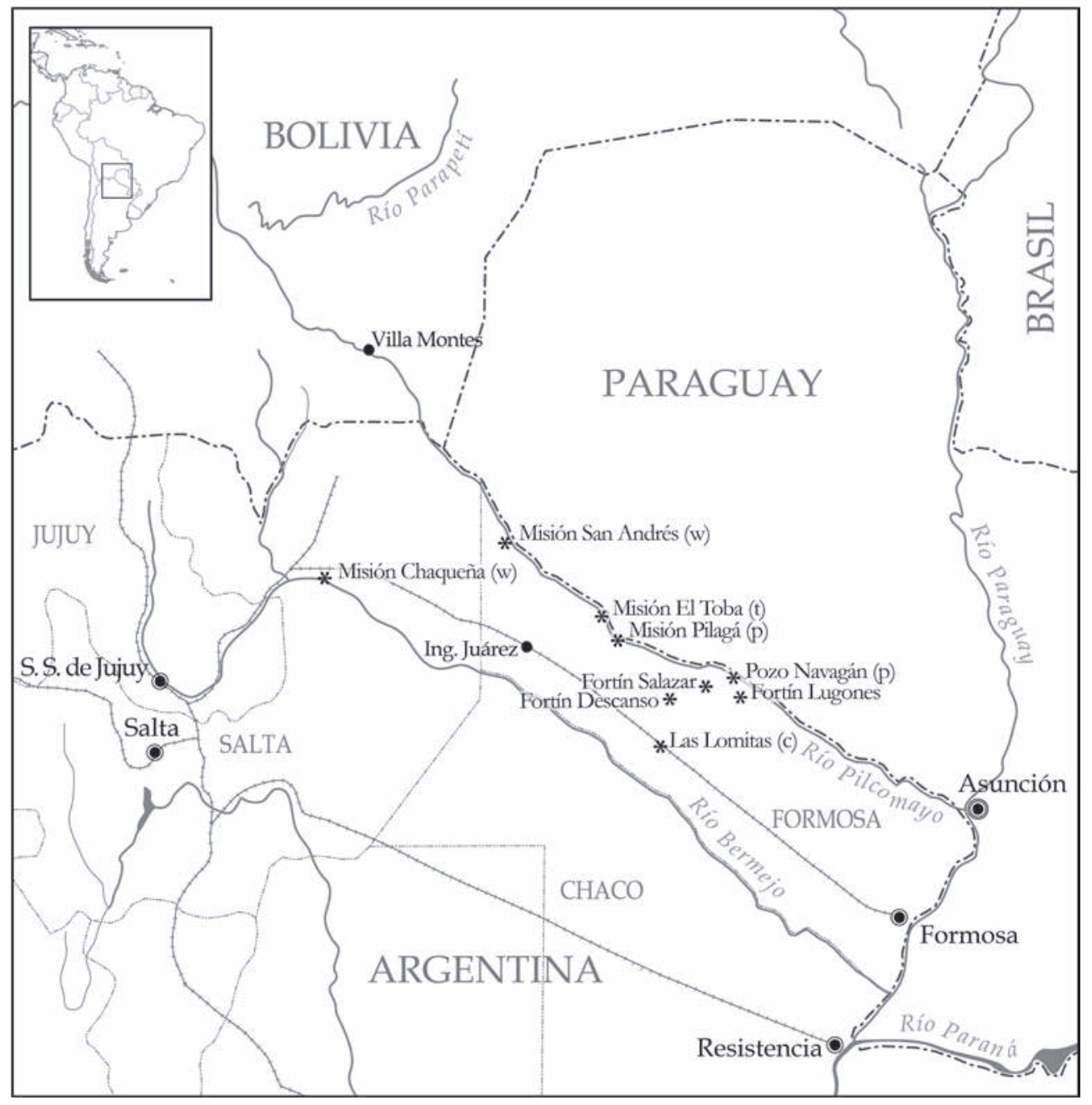

Fig. 5 - Carte des lieux visités par Métraux en 1933, élaborée par Francisco Nakayama.

Légende: (w) village wichí; (p) village pilagá; (t) village toba; (c) village criollo (créole). 


\section{Références citées}

Archives

AIA Archivo de la Iglesia Anglicana, Buenos Aires.

Yale Correspondance entre Alfred Métraux et Yvonne Oddon, General Collection, Beinecke Rare Book and Manuscript Library, Yale University, GEN MSS 350, New Haven.

FAM Fonds Alfred Métraux, Laboratoire d'anthropologie sociale, EHESS/CNRS/ Collège de France, Paris.

WUA Washington University Archives, Jules Henry Papers, 1933-1959, series 9.

\section{Bibliographie}

Amenta Sara Graciela

2008, Carlos Rodolfo Schreiter (1877-1942). Notas biográficas y epistolario de un naturalista, Centro Cultural Alberto Rougés/Fundación Miguel Lillo, Tucumán.

ARenas Pastor

2003, Etnografía y alimentación entre los toba-ñachilamole\#ek y wichi-lhuku'tas del Chaco Central (Argentina), Dunken, Buenos Aires.

BeCK Hugo H.

2005, « Una frontera de fortines. Las penosas condiciones de vida en la "Zona Militar" del Pilcomayo ", ponencia en las II Jornadas de Historia e Integración Cultural del Cono Sur, Universidad de Entre Ríos, Argentina.

BING Fernande

1964, « Entretiens avec Alfred Métraux », L’Homme, 4 (2), p. 20-32.

Bossert Federico et Diego VILLAR

2007, « La etnología chiriguano de Alfred Métraux », Journal de la société des américanistes, 93 (1), p. 127-166.

CApdevila Luc, Isabelle Combès, Nicolas Richard et Pablo Barbosa

2010, Les hommes transparents. Indiens et militaires dans la Guerre du Chaco (19321935), Presses universitaires de Rennes, Rennes.

Ceriani Cernadas César

2009, « Las enseñanzas de Don Juan Chur entre los Tobas de Formosa (Argentina, 1937-1950) », Papeles de trabajo, 2 (5), p. 1-20.

CHAQueÑo

1930, « Missionary Musings », SAMSM, 64 (716), p. 33.

Clastres Pierre

1987, « La desgracia del guerrero salvaje », in Pierre Clastres, Investigaciones en antropología política, Gedisa, Barcelona, p. 217-256.

1994, « Hommage à Alfred Métraux », Pierre Clastres, Mythologie des Indiens Chulupi, Peeters, Louvain/Paris, p. 131-135.

Comando en Jefe de las FFAA de la Nación

1950, Los partes del conductor. Comunicados oficiales sobre la Guerra del Chaco, Imprenta Militar, Asunción. 
Mission en temps de guerre

CóRdobA Lorena

2015, « Etnógrafo-misionero, misionero-etnógrafo: Alfred Métraux y John Arnott », Boletín Americanista, 70, p. 95-112.

Córdoba Lorena, Federico Bossert et Nicolas Richard

2015, Capitalismo en las selvas. Enclaves industriales en el Chaco y Amazonía indigenas (1850-1950), Ediciones del Desierto, San Pedro de Atacama.

CóRdoba Lorena et José Braunstein

2008, « Cañonazos en "La Banda”. La Guerra del Chaco y los indígenas del Pilcomayo medio », in Nicolas Richard (éd.) Mala Guerra. Los indígenas en la Guerra del Chaco 1932-1935, Servilibro/Museo del Barro, Asunción/Colibris, Paris, p. 125-147.

DA Rocha Mayor Alberto

1937, Tierra de Esteros. Relatos de los fortines chaqueños, Editorial Aniceto López, Buenos Aires.

Debaene Vincent

2014, Far Afield: French anthropology between science and literature. The University of Chicago Press, Chicago.

De la Cruz Luis M.

1995, « Qomlajépi naleua, nuestra tierra. Los sitios que contienen la tierra que da vida a los tobas de Sombrero Negro », Hacia una Carta Étnica del Gran Chaco, 6, p. 69-114.

DiJour Elizabeth

1933, «Les cérémonies d'expulsion des maladies chez les Mataco », Journal de la société des américanistes, 25 (2), p. 211-240.

Gómez Cecilia

2008, « Na'qaiq, lakawa'hegem y dapi'chi. Huellas de una zona de frontera en dos representaciones celestes toba-pilagá », Estudios latinoamericanos, 28, p. 185-209.

2010, « Los tobas del oeste formoseño y los misioneros de la South American Missionary Society », Archivos. Departamento de Antropología Cultural, 8, p. 83-119.

2011, «El hombre y la mujer: apuntes sobre la organización social toba y su relación con el ámbito celeste », Suplemento antropológico, 47 (2), p. 7-111.

GómEz Mariana

2011, De recolectoras a artesanas: género, identidades y espacialidades entre las mujeres tobas, thèse de doctorat, Anthropologie, Universidad de Buenos Aires, Buenos Aires.

GordiLlo Gastón

1999a, The bush, the plantations, and the "Devils »: culture and historical experience in the Argentinean Chaco, thèse de doctorat, Anthropologie, Universidad de Toronto, Toronto.

1999b, « A kind of sanctuary. Conversion, ambiguity, and contention at misión El Toba », trabajo presentado en la Conferencia internacional Indigenous peoples of the Chaco, christian missions, and the Nation-state, Universidad de San Andrés, Escocia.

Gordillo Gastón

2002, « Remembering “The Ancient Ones.” Memory, hegemony, and the shadows of state terror in the Argentinean Chaco ", in Winnie Lem et Belinda Leach (éd.), 
Culture, economy, power: anthropology as critique, anthropology as praxis, SUNY Press, Albany, p. 177-190.

LAURIĖRE Christine

2005, Compte rendu de « Nostalgie du néolithique. De Lausanne à Las Lomitas. Documents sur Alfred Métraux, ethnologue », Gradhiva, 1, p. 268-269.

2008, Paul Rivet, le savant et le politique, Publications scientifiques du Muséum national d'histoire naturelle (Archives), Paris.

LEAKE Alfred

1933a, « The story of the Toba mission », SAMSM, 67 (754), p. 67-69.

1933b, « The warlike ways of Toba women », SAMSM, 67 (758), p. 114-116.

1937, « Misión El Toba », SAMSM, 71 (805), p. 127-128.

LEAKE David

1967, « Breve historia de las misiones de la Iglesia Anglicana en el norte argentino », Pensamiento crítico, 55, p. 168-175.

LunT Roberto

2011, Cien años de la misión anglicana en el norte argentino. 1911-2011. Un motivo para celebrar, Diócesis de la Iglesia Anglicana en el Norte Argentino, Formosa.

Mendoza Marcela

2002, Band mobility and leadership among the Western Toba hunter-gatherers of Gran Chaco in Argentina, Edwin Mellen Press (Mellen Studies in Anthropology, 7), Lewiston.

MÉTRAux Alfred

1930, «Expedición arqueológica a La Candelaria (Provincia de Salta)», Journal de la société des américanistes, 22 (2), p. 402-404.

1933a, « La guerra primitiva en el Chaco », La Prensa, 8 de enero de 1933, Buenos Aires.

1933b, «La obra de las misiones inglesas en el Chaco », Journal de la société des américanistes, 25 (1), p. 205-209.

1933c « A remarkable testimony and appeal from an Argentine scientist », SAMSM, 67 (755), p. 79-80.

1933d, « Nouvelles de la mission A. Métraux », Journal de la société des américanistes, 25 (1), p. 203-205.

1934, « A valuable testimony to our Chaco missions », SAMSM, 68 (762), p. 15-16.

1937, «Études d'ethnographie toba-pilaga (Gran Chaco)», Anthropos, 32, p. 171-194, 378-401.

1967, Religions et magies indiennes d'Amériques du Sud, Gallimard, Paris.

1978, Itinéraires, 1. Carnets de notes et journaux de voyages (1935-1953), Payot, Paris.

2013 [1937], « Les Indiens Toba et la guerre du Chaco », in Mickaël Brohan, JeanPierre Goulard, Patrick Menget et Nathalie Pétesch (éd.), Écrits d'Amazonie.

Cosmologies, rituels, guerre et chamanisme, CNRS Éditions, Paris, p. 429-434.

NoRdENSKIÖLd Erland

2002 [1912], La vida de los Indios. El Gran Chaco (Sudamérica), APCOB, La Paz. 
Perilli de Colombres Garmendia Elena

2006, « Alfred Métraux y la Universidad Nacional de Tucumán », in Actas del I Congreso sobre la Historia de la Universidad Nacional de Tucumán, Universidad Nacional de Tucumán, Tucumán, p. 145-153.

RichARD Nicolas (éd.)

2008, Mala Guerra. Los indígenas en la Guerra del Chaco 1932-1935, Servilibro/ Museo del Barro, Asunción/Colibris, Paris.

RicHARD Nicolas

2011, « La tragedia del mediador salvaje. En torno a tres biografías indígenas de la Guerra del Chaco », Revista de ciencias sociales, 20, p. 49-80.

SAINT Enrique

1936, « El norte argentino en auto », Revista geográfica americana, 3 (30), p. 153-180.

SENDÓn Pablo

2009, « El arte del etnógrafo. Alfred Métraux y los čipaya de Carangas de 1930-1931», Trabalhos de Antropologia e Etnologia, 49 (1-4), p. 39-55.

SмIтн Colin

1929, " Continued appeals from the tobas of Argentina », SAMSM, 63 (705), p. 43.

STERPIN Adriana

1993, « L'Espace social de la prise de scalps chez les Nivacle du Gran Chaco », Hacia una nueva carta étnica del Gran Chaco, 5, p. 129-172.

Tеввотн Thomas

1937, « Misión Pilagá », SAMSM, 71 (800), p. 84-85.

1938, « Misión El Toba. September, 1937 », SAMSM, 72 (810), p. 19-20.

TomAsini Alfredo

1990, « El sentido de la guerra entre los chulupi del Gran Chaco », Folia histórica del Nordeste, 9, p. 25-54.

Torres Fernández Patricia

2006, Proyectos, discursos y politicas misionales anglicanas en el Chaco CentroOccidental durante la primera mitad del siglo XX, thèse de licence, Anthropologie, Universidad de Buenos Aires, Buenos Aires.

VACA Juan Esteban

1938, Notas de la pasada guerra del Chaco. Informaciones de cosas vistas, oídas y vividas en la zona de operaciones, [s. e.] (Círculo Militar. Biblioteca del Oficial, 240), Buenos Aires. 
